\title{
Diagnostic and prognostic utility of PD-1 in B cell lymphomas
}

\author{
S. Muenst ${ }^{\mathrm{a}}$, S. Hoeller ${ }^{\mathrm{a}}$, N. Willi ${ }^{\mathrm{b}}$, S. Dirnhofer ${ }^{\mathrm{a}}$ and A. Tzankov ${ }^{\mathrm{a}, *}$ \\ ${ }^{\mathrm{a}}$ Institute of Pathology, University Hospital, Basel, Switzerland \\ ${ }^{\mathrm{b}}$ Institute of Pathology, Cantonal Hospital Liestal, Switzerland
}

\begin{abstract}
Aims: Programmed death-1 (PD-1) is expressed by germinal center-associated helper T-cells and acts as a negative regulator of the immune system. PD-1 is encountered on tumor cells of angioimmunoblastic T-cell lymphoma and is a postulated diagnostic marker in chronic lymphocytic leukemia (CLL/SLL). Recent data suggest prognostic importance of PD-1 in follicular lymphoma (FL). We assessed the diagnostic potential and the prognostic importance of PD-1 in B-cell lymphomas.

Methods: Distribution of PD-1+ lymphocytes in B-cell lymphomas was studied on 403 cases. Correlation with known biologic and clinical key data was performed. Prognostic cut-off scores were determined by receiver operating curve analysis.

Results: PD-1+ tumor-infiltrating lymphocytes were numerous in extranodal marginal zone lymphomas and FL. Their amount decreased from FL grade 1 to grade 3 and to FL with transformation to diffuse large B-cell lymphoma. An increased amount of PD-1 tumor-infiltrating lymphocytes above the prognostic cut-off score $(>2.8 \%)$ was a positive prognostic factor of diseasespecific survival (DSS) in FL-patients. Five percent of the studied 66 CLL/SLL cases showed unequivocal PD-1 positivity of neoplastic cells.

Conclusions: Increased number of PD-1+ tumor-infiltrating lymphocytes is associated with significantly improved DSS in FL and may be useful to predict its heterogeneous clinical behavior. PD-1 has probably limited diagnostic value for primary histopathological CLL/SLL diagnostics.
\end{abstract}

\section{Introduction}

Programmed death-1 (PD-1) is a member of the CD28 costimulatory receptor superfamily. It is expressed on a subset of thymocytes and is upregulated on activated T-cells, B-cells and myeloid cells [24,25]. PD-1 inhibits T-cell activity by providing a negative signal to T-cells in conjunction with signaling through the T-cell receptor [19]. Recent studies indicate that PD-1 functions as a negative regulator of the immune system and is important in peripheral tolerance [15]. There are at least two ligands for PD-1, PD-L1 and PDL2. PD-L-PD-1 interactions lead to cell cycle arrest in G0/G1 but do not increase cell death [25]. PD-L1 engagement of PD-1 on T-cells inhibits activation of these

${ }^{*}$ Corresponding author: PD Dr. A. Tzankov, Institute of Pathology, University Hospital Basel, Schönbeinstrasse 40, 4031 Basel, Switzerland. Tel.: +41 61 2652757; Fax: +41 61 2653194; E-mail: atzankov@uhbs.ch. cells. Engagement of PD-1 by PD-L2 inhibits T-cell receptor mediated proliferation and cytokine production by CD4+ cells [25].

We and others have shown that PD-1 is specifically expressed by germinal center-associated T-cells in reactive lymphoid tissue and shows a varying distribution in defined lymphadenopathies [10,22]. It has been shown that the distribution of PD-1+ cells in nodular lymphocyte-predominant Hodgkin lymphoma is not random but that $\mathrm{PD}-1+$ cells form rosettes around the neoplastic B cells in all analyzed cases [20,23]. It has also been shown that PD-1 is a highly specific marker for tumor cells in angioimmunoblastic T-cell lymphoma (AITL) and can serve as a diagnostic marker in this entity $[10,12,20]$. PD- 1 has also been proposed to be expressed and to serve as a diagnostic marker in chronic lymphocytic leukemia (CLL) [20]. Finally, there is evidence suggesting a prognostic importance of the amount of PD-1-positive tumor-infiltrating lymphocytes, analogous to other tumor microenvironmen- 
tal components, like FOXP3-positive tumor-infiltrating lymphocytes, in follicular lymphoma (FL) and in classical Hodgkin lymphoma [1,17,23].

We performed a large scale morphometric and clinico-pathological study to systematically characterize the distribution and the prognostic importance of PD-1-positive tumor-infiltrating lymphocytes in various B-cell lymphoma entities as well as to study the possible expression of PD-1 by B-cell lymphoma tumor cells.

\section{Materials and methods}

\subsection{Samples and tissue microarray construction}

Primary diagnostic samples of previously untreated B cell lymphoma cases ( $n=403$ ) were collected from the archives of the Institutes of Pathology at the University Hospitals of Basel and Innsbruck and the Unit of Hematopathology at the University of Bologna as well as from the Cantonal Institute of Pathology in Liestal (see Table 1 for numbers of specific entities and patients' characteristics). Paraffin blocks were selected based on availability and preservation. Clinical and follow-up data were obtained by chart reviews. Retrieval of tissue and clinical data was performed according to the regulations of the local institutional review boards and data safety laws. Except for Burkitt lymphomas $(n=7)$ and T-cell rich large B-cell lymphomas $(n=5)$, which were studied on conventional slides, all other entities were studied on tissue microarray (TMA) slides, constructed and validated as described elsewhere $[1,3,13]$. For verification of TMA observations in SLL/CLL, eight additional newly diagnosed routine SLL/CLL cases were studied on conventional full tissue sections.

\subsection{Immunohistochemistry}

Slides were stained manually for PD-1. Heatinduced (steamer) antigen retrieval in citrate buffer (pH 6.0) for 10 minutes at $120^{\circ} \mathrm{C}$ was performed and the streptavidin-biotin-peroxidase detection technique was applied with amino-ethylcarbazole as chromogen. The primary goat anti-human PD-1 polyclonal antibody (AF1086 from R\&D Systems, Germany) was diluted $1: 40$ in a $1 \%$ solution of bovine serum albumin in phosphate-buffered saline (pH7.4) and the slides were incubated over night at $4{ }^{\circ} \mathrm{C}$.

\subsection{Morphometric analysis}

The total number of PD-1-positive tumor-infiltrating lymphocytes on either the TMA spots $\left(0.283 \mathrm{~mm}^{2}\right)$ or, in cases studied on conventional slides, in one medium power field $\left(1.33 \mathrm{~mm}^{2}\right)$ was counted on immunohistochemically stained slides at $200 x$ magnification. The percentage of PD-1-positive tumor-infiltrating lymphocytes in relation to all cells was determined as well. Special attention was paid to PD-1 expression on morphologically unequivocal tumor cells, which was recorded separately. Staining quality of PD-1-positive tumor-infiltrating lymphocytes was used as a reference to define positivity of the examined B-cell lymphomas. All morphometric results were mathematically referred to $1 \mathrm{~mm}^{2}$. To assess the reproducibility of the immunohistochemical data, a comparison between the results of all cases gained by two observers (SM and AT or SH and AT) was performed (see 2.4).

\subsection{Statistics}

Statistical analysis was done using the Statistical Package of Social Sciences version 15.0 for Windows (SPSS, Chicago, IL). The degree of agreement between the quantitative immunohistochemical values was evaluated by interclass correlation coefficients, using reliability Cronbach's Alpha analysis. To obtain potential biologically-meaningful associations, optimal prognostic cut-off values were established by receiver operating characteristic (ROC)-curves plotting sensitivity versus 1-specificity [2]. The cut-off point was calculated using Youden's index (Y), since this method can be applied to find the cut-off value with the highest sensitivity and specificity when there is no a priori requirement on sensitivity and/or specificity [2]. Diseasespecific survival (DSS), defined as time to death with/on lymphoma, was analyzed by the Kaplan-Meier method and compared by the log-rank test in univariable modus and by the Cox regression analysis in multivariable modus; only variables of prognostic significance from univariable tests entered the multivariable modus. Statistical significance was defined as $p<0.05$; two-sided tests were used throughout.

\section{Results}

\subsection{Staining and morphometry}

Because intensity varies between cases due to different tissue preservation methods $[7,16]$, only the ab- 
Table 1

Basic patients' characteristics of analyzed cases

\begin{tabular}{|c|c|c|c|c|c|c|c|c|c|c|c|}
\hline \multirow[t]{2}{*}{ Entity } & \multirow[t]{2}{*}{$\mathrm{N}$} & \multirow[t]{2}{*}{ Mean age } & \multirow[t]{2}{*}{$\mathrm{M}: \mathrm{F}$} & \multicolumn{4}{|c|}{ Stage } & \multirow[t]{2}{*}{$\mathrm{N}^{\text {withfollow-up }}$} & \multicolumn{2}{|c|}{ Months of median } & \multirow[t]{2}{*}{ DWD } \\
\hline & & & & $\mathrm{I}$ & II & III & IV & & follow-up & survival & \\
\hline Burkitt lymphoma & 7 & 20 & 1.3 & \multicolumn{4}{|c|}{ unknown } & 0 & & & \\
\hline DLBCL & 184 & 63 & 1.2 & 27 & 45 & 34 & 32 & 138 & 24 & 76 & 63 \\
\hline T-cell rich large B-cell lymphoma & 5 & 61 & 1.5 & 0 & 0 & 3 & 2 & 5 & 7 & 7.2 & 4 \\
\hline DLBCL ex SLL, LPL, nodal MZL & 7 & 60 & 6 & 0 & 2 & 1 & 3 & 6 & 13 & 11 & 5 \\
\hline DLBCL ex FL & 11 & 64 & 0.6 & 1 & 5 & 1 & 2 & 9 & 56 & 62 & 6 \\
\hline FL grade 3 & 7 & 57 & 0.4 & 2 & 4 & 1 & 0 & 7 & 63 & 62 & 2 \\
\hline FL grade $1-2$ & 42 & 56 & 0.7 & 2 & 14 & 3 & 20 & 39 & 50 & not reached & 7 \\
\hline Extranodal MZL & 33 & 62 & 1.1 & 15 & 5 & 0 & 0 & 20 & 101 & not reached & 6 \\
\hline Extranodal DLBCL ex MZL & 19 & 65 & 1.4 & 7 & 6 & 0 & 0 & 13 & 31 & 84 & 6 \\
\hline Mantle cell lymphoma & 10 & 64 & 4 & 0 & 2 & 0 & 8 & 10 & 17 & 22 & 8 \\
\hline Primary mediastinal B-cell lymphoma & 20 & 29 & 0.2 & \multicolumn{4}{|c|}{ unknown } & 0 & & & \\
\hline SLL/CLL & 58 & 66 & 2.2 & \multicolumn{4}{|c|}{ not applicable } & 42 & 52 & 120 & 14 \\
\hline
\end{tabular}

DWD, death with/on disease.

Treatment was either standard or consistent with the stage/risk-adapted treatment strategies according to protocols active during the time the patients were diagnosed (1989-2003).

Table 2

Quantitative results

\begin{tabular}{|c|c|c|c|c|}
\hline Entity & $\mathrm{N}$ & Mean \pm SD PD- $1+$ TIL $/ \mathrm{mm}^{2}$ & Mean \% PD-1+ TIL & $\mathrm{N}^{\text {withPD-1+TILs>mean }}$ \\
\hline Burkitt lymphoma & 7 & $23 \pm 18$ & 0.5 & $3(43 \%)$ \\
\hline DLBCL & 184 & $27 \pm 93$ & 1.1 & $20(11 \%)$ \\
\hline T-cell rich large B-cell lymphoma & 5 & $568 \pm 291$ & 51 & $2(40 \%)$ \\
\hline DLBCL ex SLL, LPL, nodal MZL & 7 & $19 \pm 26$ & 1.5 & $5(71 \%)$ \\
\hline DLBCL ex FL & 11 & $75 \pm 107$ & 2.3 & $3(27 \%)$ \\
\hline FL grade 3 & 7 & $128 \pm 105$ & 4.5 & $2(29 \%)$ \\
\hline FL grade $1-2$ & 42 & $287 \pm 228$ & 6.5 & $7(17 \%)$ \\
\hline Extranodal MZL & 33 & $231 \pm 224$ & 4.2 & $12(36 \%)$ \\
\hline Extranodal DLBCL ex MZL & 19 & $175 \pm 155$ & 3.4 & $9(47 \%)$ \\
\hline Mantle cell lymphoma & 10 & $2 \pm 6$ & 0.04 & $1(10 \%)$ \\
\hline Primary mediastinal B-cell lymphoma & 20 & $75 \pm 103$ & 1.0 & $9(45 \%)$ \\
\hline SLL/CLL & 58 & $13 \pm 37$ & 0.2 & $15(26 \%)$ \\
\hline
\end{tabular}

TIL, tumor-infiltrating lymphocytes.

solute count of positively staining cells and not the staining intensity were considered. Only unequivocally positive cells with membranous as well as cytoplasmic staining as depicted in previous papers dealing with quantification of PD-1-positive cells were scored [10, 12,17,22,23]. PD-1-positive tumor-infiltrating lymphocyte quantification was reproducible (average interobserver correlation coefficient 0.87 ), being excellent $(\alpha=0.95)$ in instances with fewer PD-1-positive cells and $\operatorname{good}(\alpha=0.82)$ in entities with higher amounts of PD-1-positive cells. Our quantitative results are shown in Table 2. Qualitative impression of the distribution of PD-1-positive tumor-infiltrating lymphocytes in the studied B cell lymphoma entities can be extracted from Fig. 1.

In accordance with our previous study, the rate of PD1-positive tumor-infiltrating lymphocytes was high in germinal center remnants, if present [22]. The amount of PD-1-positive tumor-infiltrating lymphocytes was exceptionally low in mantle cell lymphomas, small lymphocytic lymphomas/CLL (SLL/CLL) and secondary diffuse large B-cell lymphomas (DLBCL) arising from SLL/CLL, lymphoplasmacytic lymphomas or nodal marginal zone B-cell lymphomas (MZL). Contrary to previously published results [20], the neoplastic cells in all examined SLL/CLL collected from three different institutions were negative for PD-1, except for two cases with positive immunoblasts. To verify our TMA observations in SLL/CLL, we additionally stained eight newly diagnosed routine cases for PD-1 on conventional full tissue sections, and were able to identify three cases with a weak heterogeneous expression of PD-1 (weak discontinuous membranous positivity) with a particular accentuation in paraimmunoblasts (membranous and cytoplasmic positivity) and one with moderate homogenous PD-1 staining of lymphoma cells.

PD-1-positive tumor-infiltrating lymphocyte numbers were high in FL, particularly in FL grades 1-2. The amount of PD-1-positive tumor-infiltrating lym- 

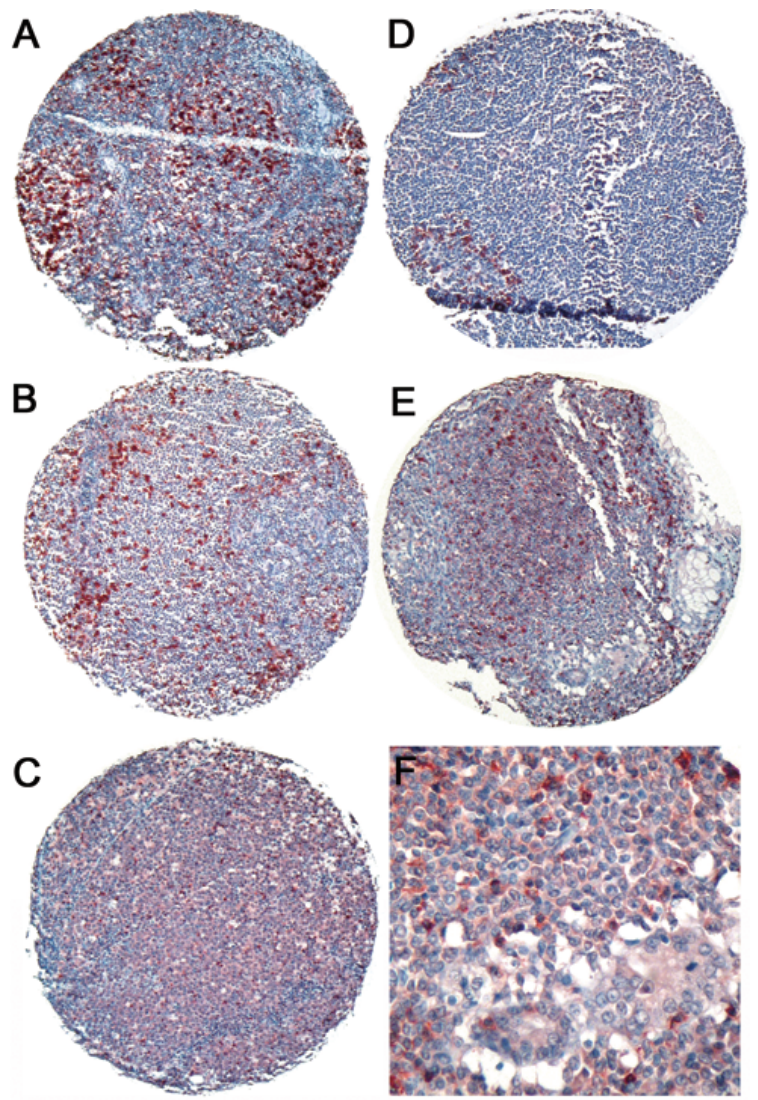

Fig. 1. Qualitative impression of the distribution of PD-1-positive tumor-infiltrating lymphocytes in the studied B-cell lymphoma entities. A. Follicular lymphoma (FL) grade 1-2 with high numbers of PD-1-positive tumor-infiltrating lymphocytes. B. FL grade 1-2 with low numbers of PD-1-positive tumor-infiltrating lymphocytes. $\mathbf{C}$. FL grade 3 with very few PD-1-positive tumor-infiltrating lymphocytes. D. Small lymphocytic B-cell lymphoma, negative for PD-1; note positive germinal center helper T-cells in a germinal center remnant. E. Marginal zone lymphoma with numerous PD-1-positive tumor-infiltrating lymphocytes; note PD-1-positive tumor-infiltrating lymphocytes between tumor cells at higher magnification $(\mathbf{F})$.

phocytes found in DLBCL arising from FL were by far higher than those found in primary DLBCL, but smaller than those found in FL grade 1-2 and 3. Extranodal MZL and extranodal DLBCL arising from extranodal MZL had higher PD-1-positive cell amounts as well. The highest amounts of PD-1-positive tumorinfiltrating lymphocytes were observed in T-cell rich large B-cell lymphomas (all cases without history of an antecedent nodular lymphocyte-predominant Hodgkin lymphoma).

PD-1 expression on neoplastic cells was observed in three primary DLBCL (all with immunoblastic morphology; two of the non-germinal center B-cell like phenotype and one of the germinal center B-cell like
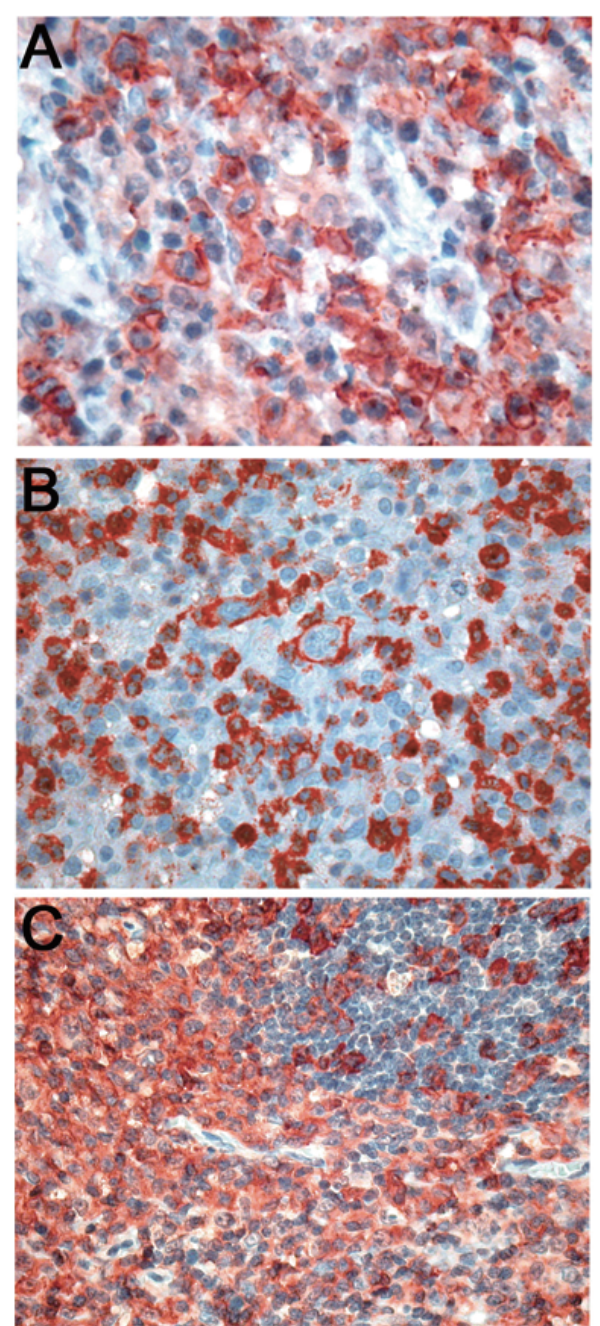

Fig. 2. PD-1 expression on neoplastic cells of (A) immunoblastic diffuse large B-cell lymphoma, (B) T-cell rich large B-cell lymphoma and (C) blast-rich extranodal marginal zone B-cell lymphoma; note intensively staining scattered small PD-1-positive tumor-infiltrating lymphocytes in B. and PD-1-positive germinal center helper T-cells in a germinal center remnant in $\mathbf{C}$.

phenotype according to Hans et al. [6]), two T-cell rich large B-cell lymphomas and three blast-rich extranodal MZL or extranodal DLBCL arising from extranodal MZL (Fig. 2).

\subsection{Determination of prognostic cut-off scores for PD-1-positive tumor-infiltrating lymphocyte numbers and survival analysis}

ROC analysis for the potential prognostic significance of PD-1-positive tumor-infiltrating lymphocyte amount among the different studied entities suggested 

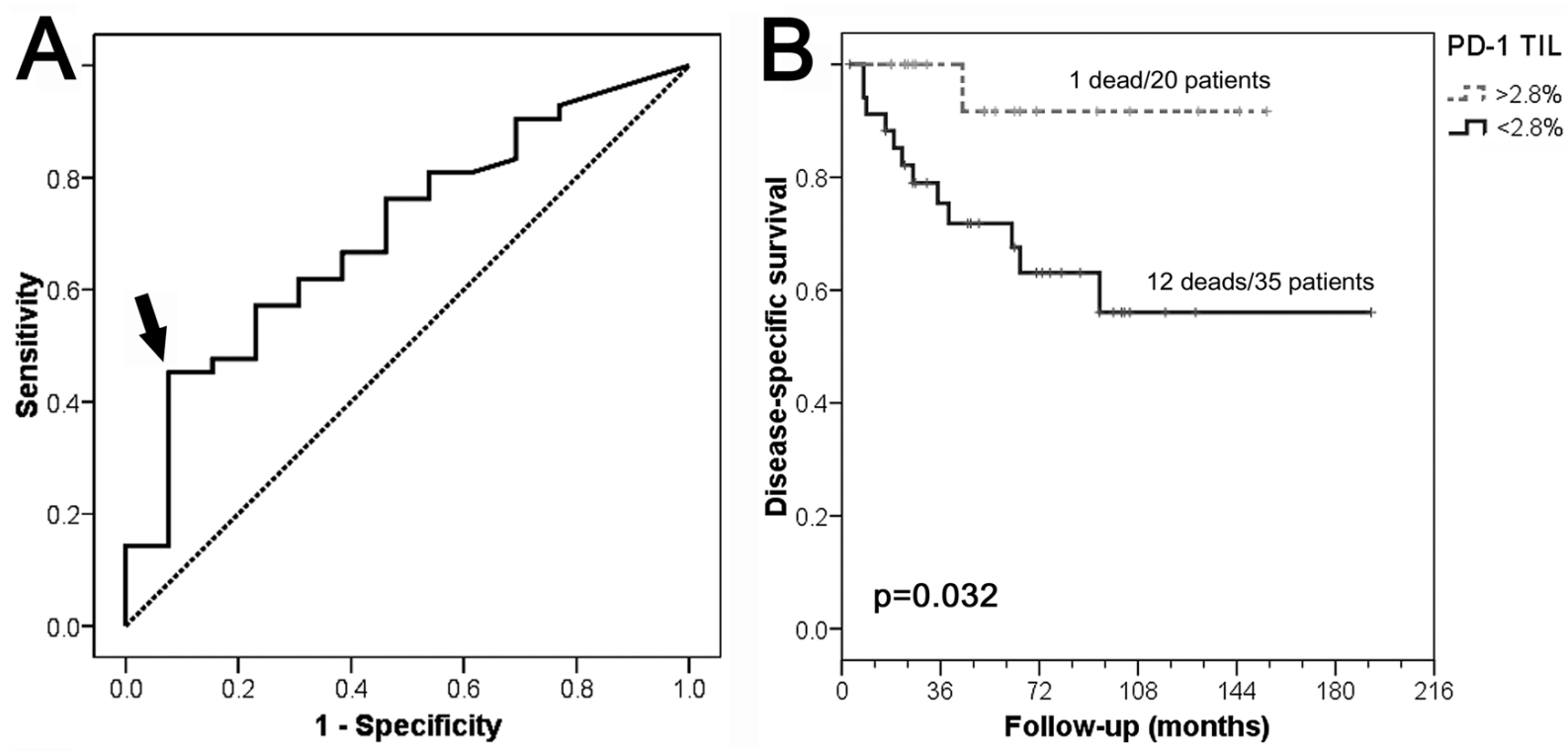

Fig. 3. Receiver operating characteristics (ROC) curve indicating a significant discriminatory potential of the amount of PD-1-positive tumor-infiltrating lymphocytes for disease-specific survival in follicular lymphomas. Note that the dark ROC curve never crosses the dotted reference line (corresponding to random guess or variable without diagnostic capability) and that the area under the ROC curve (AUROC) is 0.701 (95\% confidence interval $0.546-0.857 ; p=0.029$ ), indicating that consideration of the amount of PD-1-positive tumor-infiltrating lymphocytes is as $20 \%$ more accurate to predict survival in follicular lymphoma as a random guess (for more details see [2]). The curve point at a sensitivity of $0.45(45 \%)$ and specificity of $0.92(92 \%$; 1 -specificity $=0.08)$ is at greatest distance away from the reference line. Considering increased amount of PD-1-positive tumor-infiltrating lymphocytes, these coordinates correspond to an optimal cut-off of the amount of PD-1-positive tumor-infiltrating lymphocytes for survival prediction of $>2.8 \%$. B. Kaplan-Meier disease-specific survival curves of follicular lymphomas (grade 1-2, grade 3 and transformed ones) with respect to the amount of PD-1-positive tumor-infiltrating lymphocytes (PD-1 TIL); the p-value has been calculated by applying the log-rank test.

probable importance in FL and DLBCL arising from FL with an area under the ROC (AUROC) of 0.674 for cells/ $\mathrm{mm}^{2}$ and 0.701 for percentage (Fig. 3A); in all other studied entities, except for Burkitt- and primary mediastinal large B-cell lymphomas, for which we had no follow-up data, no prognostic role of the amount PD-1-positive tumor-infiltrating lymphocytes could be appreciated form the ROC analysis. The ROCdetermined prognostic cut-off score for the amount of PD-1-positive tumor-infiltrating lymphocytes was 168 cells $/ \mathrm{mm}^{2}$ or $2.8 \%$ (roughly, more than every 30th visible cell being stainable), respectively, with a sensitivity and specificity of $60 \%$ and $69 \%$ for the former and $45 \%$ and $92 \%$ for the latter. Using the ROCdetermined cut-off scores, the amount of PD-1-positive tumor-infiltrating lymphocytes showed a prognostic significance for DSS in FL and DLBCL arising from FL (Figure 3B). Multivariable analysis showed that elevated PD-1-positive tumor-infiltrating lymphocytes had a better, yet not significant, prognostic importance for DSS in FL than elevated FOXP3-positive tumorinfiltrating lymphocytes [1] or patient's age; stage being not a prognostic factor in this FL cohort. Cumulat- ed, high amounts of PD-1- and FOXP3-positive tumorinfiltrating lymphocytes showed a slight but not significant additive effect on DSS in FL.

\section{Discussion}

In this study we significantly expand existing quantitative and qualitative data on diagnostic and prognostic utility of PD-1-positive tumor-infiltrating lymphocyte number in B-cell lymphomas [10,20], particularly confirming their prognostic significance in FL as well as the decrease of these cells in secondary DLBCL arising from FL [17]. Our study has several advantages: (i) We used clear morphological analyses, always referring to $1 \mathrm{~mm}^{2}$, independent from the size of the individual field analyzed; (ii) The standardized approach using TMA ensured consistency in the area of tissue being considered; (iii) Sampling of tissue for TMA cores was performed independent of PD-1 staining, ensuring that there was no preferential treatment of PD-1 hot spots; (iv) All TMA were stained within one procedure, minimizing the probability of staining biases; (v) ROC 
curves were used for prognostic cut-off value determination of PD-1-positive cell density instead of arbitrary cut-off scores.

The diagnostic significance of PD-1-positive tumorinfiltrating lymphocytes in lymphoma background infiltrate has been demonstrated for different lymphoma entities $[17,23]$. We also showed a varying distribution of PD-1-positive cells in defined lymphadenopathies, which could indicate a functional relevance of these cells in such instances as well [22]. Analysis of PD-1-positive tumor-infiltrating lymphocytes is diagnostically helpful in nodular lymphocyte-predominant Hodgkin lymphoma (rosetting of PD-1-positive tumorinfiltrating lymphocytes around neoplastic cells) and might also be diagnostically important in classical Hodgkin lymphoma and in T-cell rich large B-cell lymphoma $[5,10,23]$. On average, we found two out of 58 (3\%) PD-1-positive SLL/CLL cases on our TMA as well as no PD-1 expression on tumor cells of mantle cell lymphomas, which was verified for SLL/CLL on eight additional newly diagnosed routine cases, stained for PD-1 on conventional full tissue sections. Among the latter eight cases we were able to identify three with a weak heterogeneous expression of PD-1 and one $(13 \%)$ with moderate homogenous PD-1 staining on lymphoma cells. Thus, from the histopathological point of view, staining of PD-1 for diagnosis of SLL/CLL seems not sensitive enough to be used in first run antibody panels. On the other hand, considering previous data [20] and our observed PD-1-positivity only in isolated MZL cases, expression of PD-1 among other "small B-cell lymphomas" seems to be rather specific for CLL/SLL and its evaluation might be of differential diagnostic value in occasional instances. The discrepancy of our results to those of Xerri et al. [20] could be based on differences of applied antibodies, methodology of detection, staining specificity and sensitivity, especially with regard to the definition of "positivity" and "negativity", between the studies. In at least one comparable study, Dorfman et al. were likewise unable to substantiate PD-1-positivity of neoplastic cells in SLL/CLL [10]. Our analysis showed, similarly to others, $[12,20]$ three primary DLBCL, two T-cell rich large B-cell lymphomas and three blast-rich extranodal MZL or extranodal DLBCL arising from extranodal MZL with PD-1 expression on neoplastic lymphoma cells. Although this is a small number of cases, it underlines the fact that while PD-1 is highly specific for tumor cells in AITL, its specificity is not absolute and could be rarely observed in "blast-rich" B-cell lymphomas. This should be taken into consideration in diagnostically difficult cases e.g. of AITL versus T-cell rich large B-cell lymphoma. With respect to the potential biological importance of such PD-1 expression by tumor cells, the fact that by interaction with PD-L1 and PD-L2 these tumor cells will become able to suppress other immunoreactive cells in their microenvironment should be considered. Physiologically, PD-1 acting as a potent co-inhibitor in the regulation of $\mathrm{T}$ and B-cells [19,24,25].

In agreement with the literature, the amount of PD-1positive tumor-infiltrating lymphocytes decreased from our FL grade 1 cases to secondary DLBCL arising from FL [17]. A possible explanation for this phenomenon could be that lower numbers of immunoinhibitory PD1-positive cells in FL could lead to a more stimulated immune microenvironment that in turn alleviates growth and progression of tumor cells $[1,17]$. Supporting this theory, gene expression data show that FL with poor response to anti-CD20 therapy and poor prognosis FL transforming to DLBCL within three years, are both characterized by an active immune response including high activation state of the T-cells $[4,8]$. Furthermore, neoplastic cells in FL necessitate the contact of T-cells and T-cell derived cytokines such as interleukin (IL)-4 or CLXCL13, another ligand typically expressed by germinal center-associated, PD-1-positive, helper T-cells for their proliferation $[9,11,14,18]$. This microenvironmental dependence in FL is known to be lost with progression [8]. These tumor-infiltrating lymphocytes are probably able to influence tumor behavior, which is also reflected by the improved DSS in FL and secondary DLBCL arising from FL with higher amounts of PD-1-positive tumor-infiltrating lymphocytes. Our observations are in accordance with the study by Carreras et al., showing that high numbers of tumor-infiltrating PD-1-postive cells were associated with improved progression-free survival as well as overall survival in FL [17].

In summary, the amount of PD-1-positive tumorinfiltrating lymphocytes is opposite to biological aggressiveness in FL and may be useful to prognosticate its heterogeneous clinical behavior. Our results suggest that expression of PD-1 is not reliable enough for primary histopathological CLL/SLL diagnostics and it might be occasionally encountered in tumor cells of other, particularly blast-rich, B-cell lymphomas.

\section{References}

[1] A. Tzankov, C. Meier, P. Hirschmann, P. Went, S.A. Pileri and S. Dirnhofer, Correlation of high numbers of intratumoral 
FOXP3+ regulatory $\mathrm{T}$ cells with improved survival in germinal center-like diffuse large B-cell lymphoma, follicular lymphoma and classical Hodgkin's lymphoma, Haematologica 93 (2008), 193-200.

[2] A. Tzankov, I. Zlobec, P. Went, H. Robl, S. Hoeller and S. Dirnhofer, Prognostic immunophenotypic biomarker studies in diffuse large B cell lymphoma with special emphasis on rational determination of cut-off scores, Leuk Lymphoma $\mathbf{5 1}$ (2010), 199-212.

[3] A. Tzankov, P. Went, A. Zimpfer and S. Dirnhofer, Tissue microarray technology: principles, pitfalls and perspectives lessons learned from hematological malignancies, Exp Gerontol 40 (2005), 737-744.

[4] A.M. Glas, L. Knoops, L. Delahaye et al., Gene-expression and immunohistochemical study of specific T-cell subsets and accessory cell types in the transformation and prognosis of follicular lymphoma, J Clin Oncol 25 (2007), 390-398.

[5] B. Chetaille, F. Bertucci, P. Finetti et al., Molecular profiling of classical Hodgkin lymphoma tissues uncovers variations in the tumor microenvironment and correlations with EBV infection and outcome, Blood 113 (2009), 2765-3775.

[6] C.P. Hans, D.D. Weisenburger, T.C. Greiner et al., Confirmation of the molecular classification of diffuse large B-cell lymphoma by immunohistochemistry using a tissue microarray, Blood 103 (2004), 275-282.

[7] D. Atkins, K. Reiffen, C.L. Tegtmeier, H. Winther, M.S. Bonato and S. Storkel, Immunohistochemical detection of EGFR in paraffin-embedded tumor tissues: variation in staining intensity due to choice of fixative and storage time of tissue sections, J Histochem Cytochem 52 (2004), 893-901.

[8] D. de Jong, Molecular pathogenesis of follicular lymphoma: a cross talk of genetic and immunologic factors, J Clin Oncol 10 (2005), 6358-6663.

[9] D. Schmitter, M. Koss, E. Niederer, R.A. Stahel and G. Pichert, T-cell derived cytokines co-stimulate proliferation of CD40activated germinal centre as well as follicular lymphoma cells, Hematol Oncol 15 (1997), 197-207.

[10] D.M. Dorfman, J.A. Brown, A. Shahsafaei and G.J. Freeman, Programmed death-1 (PD-1) is a marker of germinal centerassociated $\mathrm{T}$ cells and angioimmunoblastic T-cell lymphoma, Am J Surg Pathol 30 (2006), 802-810.

[11] D.T. Umetsu, L. Esserman, T.A. Donlon, R.H. DeKruyff and R. Levy, Induction of proliferation of human follicular (B type) lymphoma cells by cognate interaction with $\mathrm{CD} 4+\mathrm{T}$ cell clones, J Immunol 144 (1990), 2550-2557.

[12] G. Roncador, J.F. Garcia Verdes-Montenegro, S. Tedoldi et al., Expression of two markers of germinal center T cells (SAP and PD-1) in angioimmunoblastic T-cell lymphoma, Haematologica 92 (2007), 1059-1066.

[13] H. Adams, P. Liebisch, P. Schmid, S. Dirnhofer and A. Tzankov, Diagnostic utility of the B-cell lineage markers
CD20, CD79a, PAX5, and CD19 in paraffin-embedded tissues from lymphoid neoplasms, Appl Immunohistochem Mol Morphol 17 (2009), 96-101.

[14] H. Husson, A.S. Freedman, A.A. Cardoso et al., CXCL13 (BCA-1) is produced by follicular lymphoma cells: role in the accumulation of malignant B cells, Br J Haematol 119 (2002), 492-495.

[15] H.C. Probst, K. McCoy, T. Okazaki, T. Honjo and M. van den Broek, Resting dendritic cells induce peripheral CD8+ T cell tolerance through PD-1 and CTLA-1, Nat Immunol 6 (2005), 280-286.

[16] I. Zlobec, L. Terracciano, J.R. Jass and A. Lugli, Value of staining intensity in the interpretation of immunohistochemistry for tumor markers in colorectal cancer, Virchows Arch 451 (2007), 763-769.

[17] J. Carreras, A. Lopez-Guillermo, G. Roncador et al., High numbers of tumor-infiltrating programmed cell death 1positive regulatory lymphocytes are associated with improved overall survival in follicular lymphoma, J Clin Oncol $\mathbf{2 7}$ (2009), 1470-1476.

[18] K.R. Calvo, B. Dabir, A. Kovach, C. Devor, R. Bandle, A. Bond, J.H. Shih and E.S. Jaffe, IL-4 protein expression and basal activation of Erk in vivo in follicular lymphoma, Blood 112 (2008), 3818-3826.

[19] L. Chen, Co-inhibitory molecules of the B7-CD28 family in the control of T-cell immunity, Nat Rev Immunol 4 (2004), $336-347$.

[20] L. Xerri, B. Chetaille, N. Seriari, C. Attias, Y. Guillaume, C. Arnoulet and D. Olive, Programmed death 1 is a marker of angioimmunoblastic T-cell lymphoma and B-cell small lymphocytic lymphoma/chronic lymphocytic leukemia, Hum Pathol 39 (2008), 1050-1058.

[21] S. Inoges, A. Lopez-Diaz de Cerio, N. Zabalegui et al., Prolonged idiotypic vaccination against follicular lymphoma, Leuk Lymphoma 50 (2009), 47-53.

[22] S. Muenst, S. Dirnhofer and A. Tzankov, Distribution of PD$1+$ lymphocytes in reactive lymphadenopathies, Pathobiology 77 (2010), 24-27.

[23] S. Muenst, S. Hoeller, S. Dirnhofer and A. Tzankov, Increased programmed death-1 tumor-infiltrating lymphocytes in classical Hodgkin lymphoma substantiate reduced overall survival, Hum Pathol 40 (2009), 1715-1722.

[24] Y. Kitazawa, M. Fujino, Q. Wang, H. Kimura, M. Azuma, M. Kubo, R. Abe and X. Li, Involvement of the programmed death-1/programmed death-1 ligand pathway in CD4+CD25+ regulatory T-cell activity to suppress alloimmune responses, Transplantation 83 (2007), 774-782.

[25] Y. Lachmann, C.R. Wood, T. Chernova et al., PD-L2 is a second ligand for PD-1 and inhibits T cell activation, Nat Immunol 2 (2001), 261-268. 


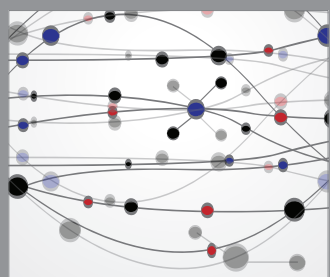

The Scientific World Journal
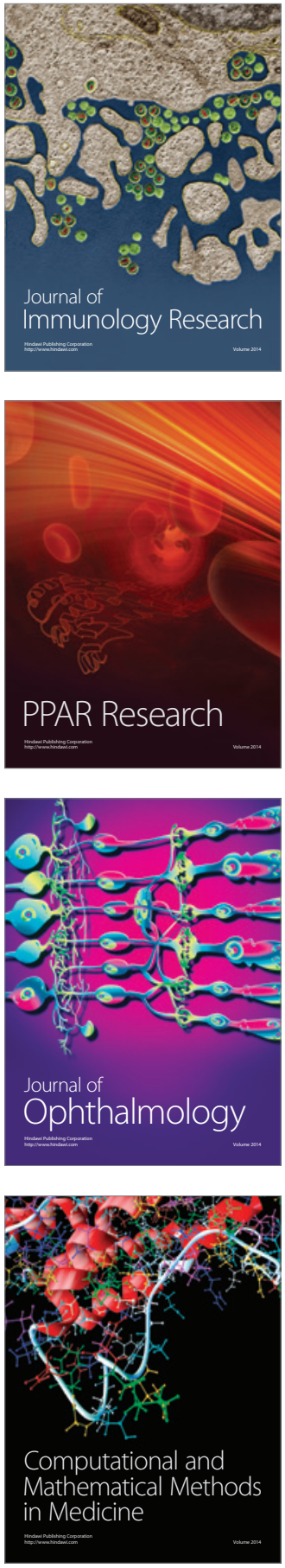

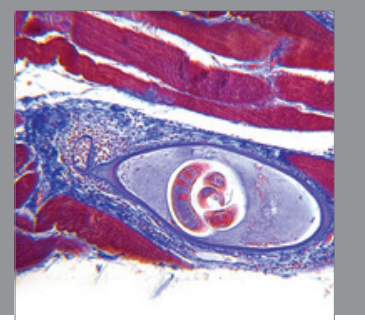

Gastroenterology

Research and Practice
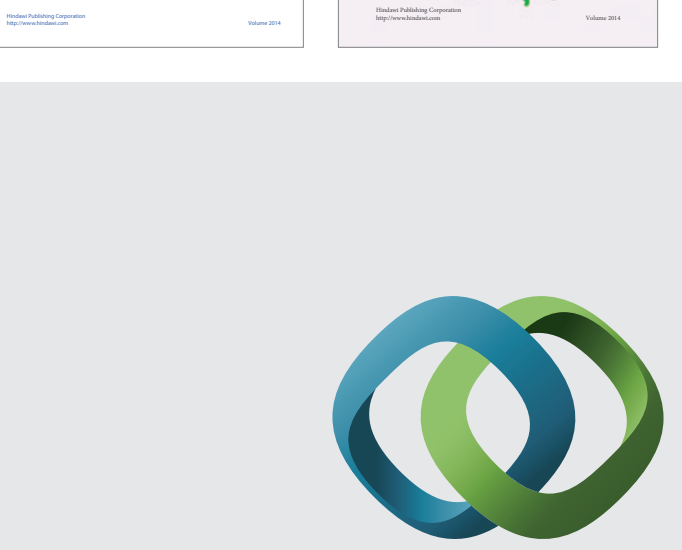

\section{Hindawi}

Submit your manuscripts at

http://www.hindawi.com
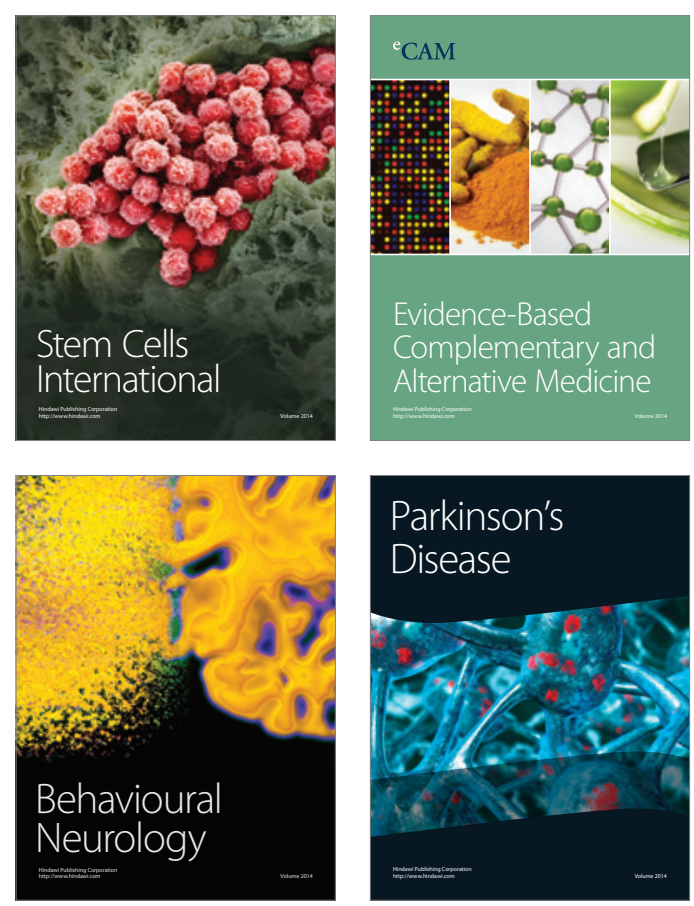

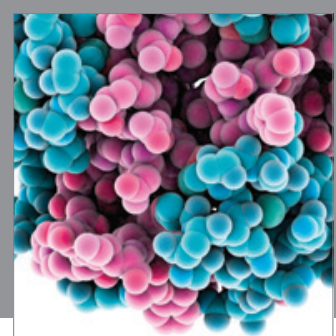

Journal of
Diabetes Research

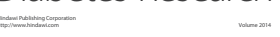

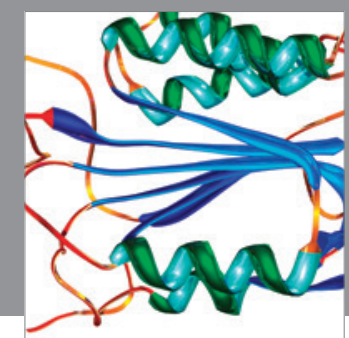

Disease Markers
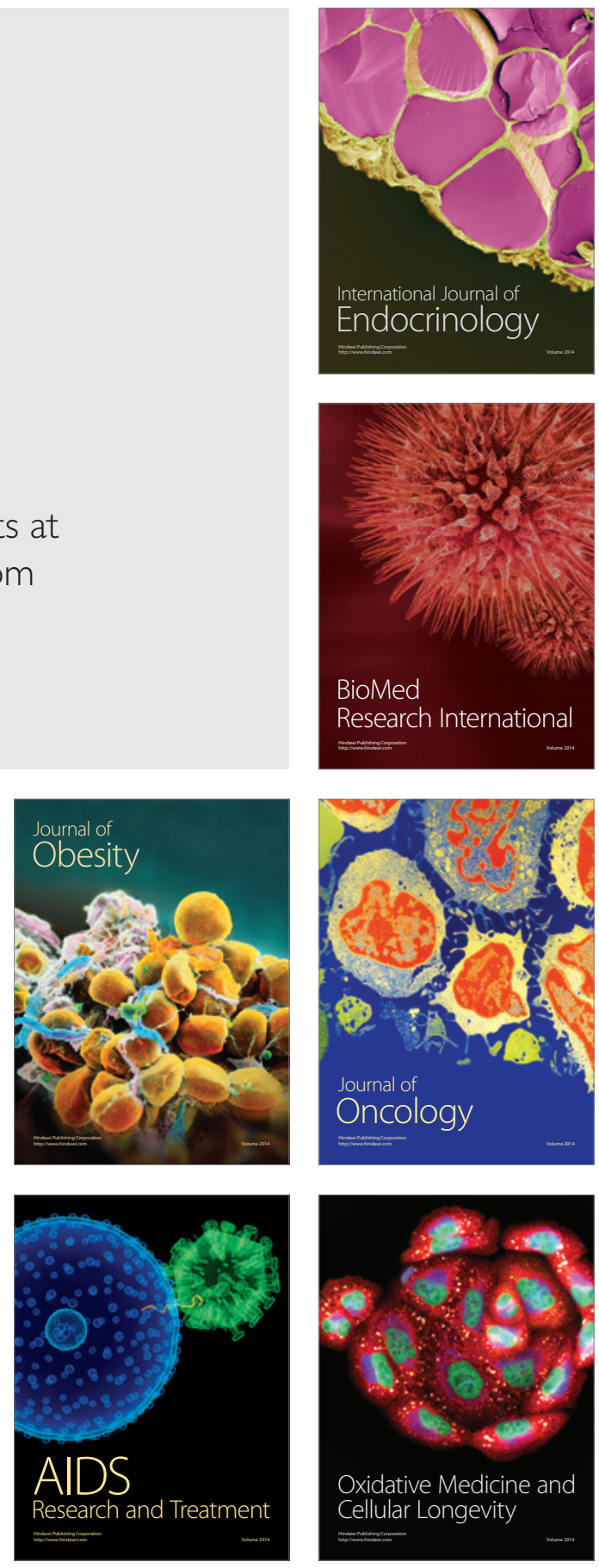